Review Article

\title{
Review of Ultrafast Demagnetization After Femtosecond Laser Pulses: A Complex Interaction of Light with Quantum Matter
}

\author{
Manfred Fähnle ${ }^{1, \text { *, Michael Haag }}{ }^{1}$, Christian Illg ${ }^{1}$, Benedikt Mueller ${ }^{1}$, Weikai Weng ${ }^{1}$, \\ Theodoros Tsatsoulis ${ }^{1}$, Haonan Huang ${ }^{1}$, Johan Briones ${ }^{1}$, Nicolas Teeny ${ }^{1}$, Lifa Zhang ${ }^{2}$, \\ Tilmann Kuhn ${ }^{3}$ \\ ${ }^{1}$ Max Plank Institute for Intelligent Systems, Stuttgart, Germany \\ ${ }^{2}$ Department of Physics and Institute of Theoretical Physics, Nanjing Normal University, Nanjing, China \\ ${ }^{3}$ Institute of Solid State Theory, University of Münster, Münster, Germany
}

Email address:

faehnle@is.mpg.de (M. Fähnle), zhanglf@njnu.edu.cn (L. Zhang), Tilmann.Kuhn@uni-muenster.de (T. Kuhn)

${ }^{*}$ Corresponding author

\section{To cite this article:}

Manfred Fähnle, Michael Haag, Christian Illg, Benedikt Mueller, Weikai Weng, Theodoros Tsatsoulis, Haonan Huang, Johan Briones, Nicolas Teeny, Lifa Zhang, Tilmann Kuhn. Review of Ultrafast Demagnetization After Femtosecond Laser Pulses: A Complex Interaction of Light with Quantum Matter. American Journal of Modern Physics. Vol. 7, No. 2, 2018, pp. 68-74. doi: 10.11648/j.ajmp.20180702.12

Received: December 27, 2017; Accepted: January 9, 2018; Published: January 18, 2018

\begin{abstract}
When a magnetic film is excited by a femtosecond laser pulse, either with THz or with optical frequencies, then there is at least a partial demagnetization within a few hundred femtoseconds, followed by a remagnetization to the original state on a bit longer time scale. This phenomenon is caused by a complex interaction of light with quantum matter. This paper gives a review of the present knowledge of the underlying physics. It discusses first the situation of a direct change of the magnetization by its interaction with the electromagnetic wave of the laser pulse, which appears during $\mathrm{THz}$ laser pulses with small field amplitudes. Then it considers the situation of an indirect change which appears after THz laser pulses with large field amplitudes and after optical laser pulses. In these cases the laser photons primarily excite electrons, with subsequent modifications of their spin-angular momenta by spin-flip scatterings of these electrons at quasiparticles, either at other electrons or at phonons or at magnons. The contributions of these various spin-flip scatterings to demagnetization are investigated. Then the transfer of angular momentum from the electronic spin system to the lattice during ultrafast demagnetization is discussed by describing the lattice vibrations in terms of magnetoelastic spin-phonon modes. Finally, the effect of electronic correlations in the sense of the density-matrix theory is investigated.
\end{abstract}

Keywords: Ultrafast Demagnetization, Femtosecond Laser Pulses, THz and Optical Frequencies, Magnetization Precession, Spin-Flip Scatterings of Electrons, Angular Momentum Transfer, Electronic Correlations

\section{Introduction}

When a magnetic film on a substrate is excited by a femtosecond (fs) laser pulse, either with THz or with optical frequencies, then there is at least a partial demagnetization within a few hundred fs, followed by a remagnetization to the original state on a bit longer time scale [1], as sketched in Figure 1. At present this is the fastest possible macroscopic manipulation of the magnetization of a film, and therefore it is of great interest from a fundamental point of view and from the view of a possible application in magnetic computers. It is closely related to the all-optical switching [2], where the magnetization of a ferrimagnet is inverted by the action of a fs optical laser pulse.

The phenomenon of ultrafast de-and remagnetization has been investigated since its discovery [3] worldwide very intensively both experimentally and theoretically, and it is still under heavy discussion. The reason is that it is caused by 
a complex interaction of light with quantum matter, and that the theoretical description requires very sophisticated methods. There may be different mechanisms contributing to the demagnetization. First, there may be a direct change of the magnetization by its interaction with the electromagnetic wave of the laser pulse. This is the case during $\mathrm{THz}$ laser pulses with small field amplitudes (section 2). Second, there may be an indirect change (section 3). This is the case after $\mathrm{THz}$ laser pulses with large field amplitudes and after optical laser pulses. In this case the laser photons primarily excite electrons, with subsequent modifications of their spin-angular momenta by spin-flip scatterings of the electrons at quasiparticles, either at other electrons (section 3.1), at phonons (section 3.2) or at magnons (section 3.3). Third, if the magnetic film is on a conducting substrate, then there may be a contribution of the so-called superdiffusion [4] to the demagnetization. Because most experiments have been performed for nonconducting substrates, superdiffusion is not discussed in this review. In the context of spin-flip scatterings of electrons at quasiparticles we will also discuss two general issues, the transfer of angular momentum from the electronic spin system to the lattice (section 4) and the effect of electronic correlations in the sense of a density-matrix theory (section 5). Conclusions will be given in section 6 .

\section{normalized magnetization}

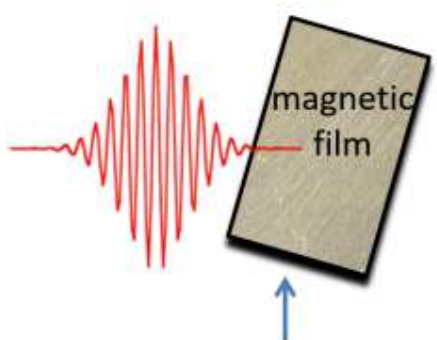

substrate

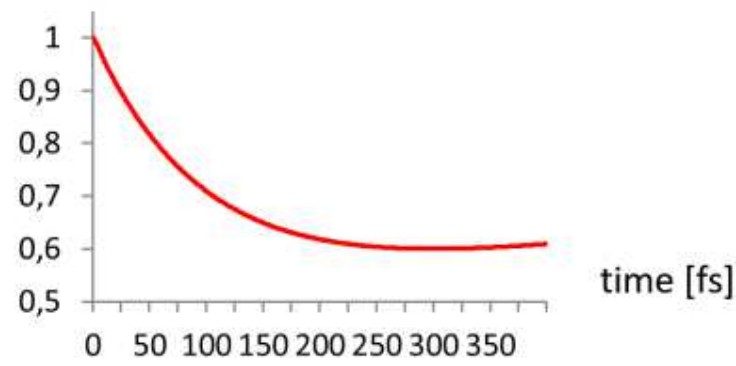

Figure 1. Sketch of the ultrafast de-and remagnetization after $f_{s}$ laser pulses.

\section{Direct Change of the Magnetization}

First the direct change of the magnetization by its interaction with the electromagnetic wave of the laser pulse which appears during $\mathrm{THz}$ laser pulses with small field amplitudes [5] is discussed. There there is a coherent interaction between the magnetization $M(t)$ and the effective field $B_{\text {eff }}(t)$ which oscillates in time due to the action of the electromagnetic wave of the laser pulse. This leads to a direct correlation between the temporal $\mathrm{M}(\mathrm{t})$ and the temporal oscillation of the laser field, in contrast to the lack of such a direct correlation after $\mathrm{THz}$ laser pulses with large field amplitudes and after optical laser pulses (section 3 ). The direct correlation leads to a precessional motion of $\mathrm{M}(\mathrm{t})$ due to the Zeeman torque exerted by $\mathrm{B}_{\text {eff }}(\mathrm{t})$ on $\mathrm{M}(\mathrm{t})$, which is proportional to $\mathrm{M}(\mathrm{t}) \times \mathrm{B}_{\text {eff }}(\mathrm{t})$. This precessional motion is observable after $\mathrm{THz}$ laser pulses with small field amplitudes because of the lack of a strong heating of the sample. In fact, $\mathrm{THz}$ photon energies are about three orders of magnitude smaller than optical photon energies, and this avoids a substantive heating of the sample. Therefore we can neglect spin-flip scatterings of hot excited electrons which appear after $\mathrm{THz}$ laser pulses with large field amplitudes and after optical laser pulses (section 3 ). They would change $B_{\text {eff }}(t)$ incoherently in time, and this would lead to a dephasing of the magnetization dynamics, so that no precessional motion would be observable. However, it is observable during $\mathrm{THz}$ laser pulses with small field amplitudes.

The precessional motion leads to a demagnetization of the film during the action of the laser pulse. After the action there is a slow recovery of the original state, because in these experiments a constant external magnetic field is applied, which exerts a torque on $\mathrm{M}(\mathrm{t})$, leading to a damped magnetization dynamics back to the original state.

\section{Indirect Change of the Magnetization}

Now the indirect change of the magnetization which occurs after $\mathrm{THz}$ laser pulses with large field amplitudes and after optical laser pulses is discussed. In these cases the laser photons primarily excite electrons, with subsequent modifications of their spin angular momenta by spin-flip scatterings of the electrons at quasiparticles, either other electrons (section 3.1), phonons (section 3.2) or magnons (section 3.3). These spin-flip scatterings change $B_{\text {eff }}(t)$ incoherently in time, as discussed in section 2, which leads to a dephasing of the magnetization dynamics so that no precessional motion is observable. The spin-flip scatterings change the magnetization in time and lead to a demagnetizazion, because the spin-flip scattering rates for scatterings from spin-up to spin-down and for scatterings from spin-down to spin-up are different.

It has been shown experimentally [1] that the by far dominant part of the demagnetization takes place after, not during the action of the laser pulse, so that the magnetic moment $\mathrm{m}$ of the system directly after the action of the laser pulse is still the equilibrium moment $\mathrm{m}_{0}$ which one had before the action of the laser pulse, at the temperature at which the experiment is performed. In principle the effect of the laser pulse could be described directly by the interaction of the laser photons with the matter. However, in nearly all papers the effect of the laser pulse is described by the Elliott-Yafet scenario [6], which gives an ansatz for the occupation 
numbers of electronic states at the time $t_{s}$ directly after the action of the laser pulse. In a system without spin-orbit coupling the electronic state would be described by the electronic wave vector $\mathrm{k}$, the band index $\mathrm{i}$, and the spin orientation s. In realistic systems with spin-orbit coupling the spin parts of the electronic states are not pure spin states, but mixtures of an eigenstate of the spin-operator with spin-up and a second eigenstate with spin-down (see, e.g., [1]). In magnetic transition metals the electronic states have a dominant spin character, i.e., their expectation values of the spin are not identical but close to the one in a pure spin state. In the following we therefore denote this dominant spin character of the electronic state by the spin index s.

In the Elliott-Yafet scenario it is assumed that the electrons which were excited by the laser photons thermalize more or less instantly due to very rapid and frequent electron-electron scatterings via their Coulomb interactions. It is then assumed that the occupation numbers of electronic states with energies $\varepsilon_{\mathrm{ik}}^{\mathrm{s}}$ at time $\mathrm{t}_{\mathrm{s}}$ directly after the action of the laser pulse are given by nonequilibrium Fermi-Dirac distributions

$$
\mathrm{f}\left(\varepsilon_{\mathrm{ik}}^{\mathrm{s}}, \varepsilon_{\mathrm{F}}^{\mathrm{s}}\left(\mathrm{t}_{\mathrm{s}}\right)\right)=\left(\exp \left(\frac{\varepsilon_{\mathrm{ik}}^{\mathrm{s}}-\varepsilon_{\mathrm{F}}^{\mathrm{s}}\left(\mathrm{t}_{\mathrm{s}}\right)}{\mathrm{k}_{\mathrm{B}} \mathrm{T}_{\mathrm{e}}\left(\mathrm{t}_{\mathrm{s}}\right)}\right)+1\right)^{-1} .
$$

These are nonequilibrium distributions because they have two chemical potentials $\varepsilon_{\mathrm{F}}^{\mathrm{s}}\left(\mathrm{t}_{\mathrm{s}}\right)$ for the two values of $\mathrm{s}$, and an electron temperature $\mathrm{T}_{\mathrm{e}}\left(\mathrm{t}_{\mathrm{s}}\right)$, which is increased above the temperature at which the experiment is performed because of the excitation. $\mathrm{T}_{\mathrm{e}}\left(\mathrm{t}_{\mathrm{s}}\right)$ is taken from a fit of the so-called three-temperature model of ultrafast demagnetization [1] to the experimental data. It is-depending on the laser fluence and the film material-typically between $600 \mathrm{~K}$ and $1200 \mathrm{~K}$. The two chemical potentials $\varepsilon_{\mathrm{F}}^{\mathrm{s}}\left(\mathrm{t}_{\mathrm{s}}\right)$ are determined from two equations:

$$
\begin{gathered}
\mathrm{m}=\mu_{\mathrm{B}}\left(\mathrm{N}_{0}^{\uparrow}-\mathrm{N}_{0}^{\downarrow}\right)=\mathrm{m}_{0}, \\
\mathrm{~N}=\mathrm{N}_{0}^{\uparrow}+\mathrm{N}_{0}^{\downarrow}
\end{gathered}
$$

Here $\mu_{\mathrm{B}}$ is Bohr's magneton, $\mathrm{N}_{0}^{\uparrow}$ and $\mathrm{N}_{0}^{\downarrow}$ are the numbers of electrons with $s=\uparrow$ and $s=\downarrow$, which can be calculated from the nonequilibrium Fermi-Dirac distributions and from the electronic density of states for the two spin orientations, and $\mathrm{N}$ is the total number of electrons in the system. Solving (2) and (3) yields the two chemical potentials $\varepsilon_{\mathrm{F}}^{\mathrm{s}}\left(\mathrm{t}_{\mathrm{s}}\right)$. At later times, $t>t_{s}$, the actual occupation numbers change in time due to the spin-flip scattering processes, and this change is determined directly from the calculated spin-flip scattering rates.

\subsection{Electron-Electron Scatterings}

Now the contribution of electron-electron scatterings to the ultrafast demagnetization is discussed. These scatterings contribute to the change of the magnetization only in systems with spin-orbit coupling. The electron-electron scatterings are the strongest scatterings which the electrons feel, and they can be described by the screened Coulomb interaction between them. Electron-electron scatterings which contribute dominantly to the demagnetization are those for which the total spin angular momentum of the two electrons is nearly (not exactly) conserved:

$$
\begin{aligned}
& \left|\mathrm{k}_{1} \uparrow\right\rangle+\left|\mathrm{k}_{2} \downarrow\right\rangle \rightarrow\left|\mathrm{k}_{3} \uparrow\right\rangle+\left|\mathrm{k}_{4} \downarrow\right\rangle . \\
& \left|\mathrm{k}_{1} \uparrow\right\rangle+\left|\mathrm{k}_{2} \downarrow\right\rangle \rightarrow\left|\mathrm{k}_{3} \downarrow\right\rangle+\left|\mathrm{k}_{4} \uparrow\right\rangle .
\end{aligned}
$$

In systems without spin-orbit coupling these scatterings would not contribute to demagnetization, because the total spin angular momentum of the two electrons would be exactly conserved. In systems with spin-orbit coupling there are the above discussed spin-mixed states, and the spin expectation values of $\left|\mathrm{k}_{1} \uparrow\right\rangle$ and $\left|\mathrm{k}_{4} \uparrow\right\rangle$ or of $\left|\mathrm{k}_{2} \downarrow\right\rangle$ and $\left|\mathrm{k}_{4} \downarrow\right\rangle$ are in general different, so that the total spin angular momentum of the two electrons is not exactly conserved, and then the scatterings of (4) and (5) can contribute to demagnetization.

During ultrafast demagnetization the magnetization changes in time, and therefore the electronic band structure changes dynamically, i.e., the energies and the spin expectation values change. It has been shown in [7], that electron-electron scatterings yield a demagnetization comparable to the experimentally observed demagnetization only if the dynamic property of the band structure is taken into account. However, in this paper an empirical tight-binding model for the band structure was used. In [8], this result of [7] was reproduced by ab-initio calculations within the spin-density functional electron theory.

\subsection{Electron-Phonon Scatterings}

For the contribution of electron-phonon scatterings to ultrafast demagnetization we refer to [9]. The basis of the theory is an extension of the electron-phonon spin-flip scattering matrix element of Yafet [6], which was derived for paramagnets to ferromagnets. This matrix element contains spatial derivatives of the lattice potential, which is represented in the rigid-ion approximation [10]. The equilibrium potential $V^{0}\left(r ;\left\{R_{n}^{0}\right\}\right)$ with all atoms $n$ at their equilibrium positions $R_{n}^{0}$ can be decomposed in atomic potentials $\mathrm{v}_{\mathrm{n}}^{0}$ which are centered at these equilibrium positions,

$$
\mathrm{V}^{0}\left(\mathrm{r} ;\left\{\mathrm{R}_{\mathrm{n}}^{0}\right\}\right)=\sum_{\mathrm{n}} \mathrm{v}_{\mathrm{n}}^{0}\left(\mathrm{r}-\mathrm{R}_{\mathrm{n}}^{0}\right)
$$

The rigid-ion approximation says that one can still use the atomic equilibrium potentials $\mathrm{v}_{\mathrm{n}}^{0}$ to describe the potential $\mathrm{V}\left(\mathrm{r} ;\left\{\mathrm{R}_{\mathrm{n}}\right\}\right)$ of the slightly distorted lattice (e.g., by the effect of a phonon), where the lattice atom positions $R_{n}$ are displaced from their equilibrium positions,

$$
\mathrm{V}\left(\mathrm{r} ;\left\{\mathrm{R}_{\mathrm{n}}\right\}\right)=\sum_{\mathrm{n}} \mathrm{v}_{\mathrm{n}}^{0}\left(\mathrm{r}-\mathrm{R}_{\mathrm{n}}\right)
$$

The calculation of the ultrafast demagnetization after fs optical laser pulses by spin-flip electron-phonon scatterings was performed on the basis of Fermi's golden rule for time-oscillating perturbations, here given by the time-oscillating perturbations produced by the phonons. Thereby the dynamical change of the electronic band structure during demagnetization was taken into account. The phonon frequencies and polarization vectors were obtained from a force-constant model, where the force constants were 
calculated ab initio [11]. The electronic states were calculated $\mathrm{ab}$ initio by the relativistic version [12] of the linear-muffin-tin-orbital method [13] in local-spin-density approximation $[14,15]$ and in atomic-sphere approximation [13].

The electron-phonon matrix elements contain two parts, the phonon-induced modification of the lattice potential (Elliott part) and the phonon-induced modification of the spin-orbit coupling (Yafet part). In [9] it was found that both parts are similarly important. Furthermore, it was found that electron-phonon spin-flip scatterings contribute to ultrafast demagnetization, but they cannot describe the experimentally observed demagnetization quantitatively. There must be other contributions, and the strong contribution of electron-electron scatterings was discussed already in section 3.1.

\subsection{Electron-Magnon Scatterings}

Electron-magnon scatterings can lead to a change of the magnetization only in systems with spin-orbit coupling. To describe them, first an expression for the spin-flip electron-magnon scattering matrix elements had to be derived [16].

The calculations of the demagnetization by electron-magnon scatterings were performed on the basis of Fermi's golden rule for time-oscillating perturbations, here given by the time-oscillating perturbations produced by magnons. Thereby the dynamical change of the electronic band structure was taken into account. The magnon dispersions were calculated by the ab-initio spin density functional electron theory [17]. The softening of the magnons with increasing temperature was taken into account via the temperature dependence of the spin-wave stiffness. The electronic states were calculated by the methods described in section 3.2 .

Carpene et al. [18] suggested that electron-magnon scatterings increase the orbital momentum of the system and decrease the spin momentum by the emission of magnons, and that the orbital momenta of the involved electrons are immediately quenched by the crystal field.

$$
\left|\mathrm{k}_{1} \uparrow\right\rangle \rightarrow\left|\mathrm{k}_{2} \downarrow\right\rangle+\text { magnon }
$$

In a system without spin-orbit coupling there would be no change of the magnetization by this process. It was shown in [19] that the effect of mere electron-magnon scatterings is very small.

Inspired by the ideas of Manchon et al. [20], and of Schellekens and Koopmans [21], in [19] it was suggested that a combination of electron-phonon and electron-magnon scatterings may explain the experimentally observed demagnetization, because of the following reason: It was shown that the number of magnon emissions is considerably larger than the number of magnon absorptions, so that the dominant effect of electron-magnon scatterings are transitions from spin-down to spin-up,

$$
\left|\mathrm{k}_{1} \downarrow\right\rangle \rightarrow\left|\mathrm{k}_{2} \uparrow\right\rangle+\text { magnon. }
$$

The number of spin-flip scatterings by phonons which decrease the magnetic moment of the system (demagnetizing electron-phonon scatterings), i.e., those from spin-up to spin-down,

$$
\left|\mathrm{k}_{1} \uparrow\right\rangle \rightarrow\left|\mathrm{k}_{2} \downarrow\right\rangle \pm \text { phonon }
$$

is only slightly larger than the number of scatterings which increase the magnetic moment, i.e., those from spin-down to spin-up. Furthermore, after the scatterings of type (10) the number of spin-up states which can be used for further demagnetizing electron-phonon scatterings is reduced. Because of these two points mere electron-phonon scatterings cannot explain the experimentally observed demagnetization. However, as shown above the dominant effect of electron-magnon scatterings are transitions from spin-down to spin-up, i.e., they increase the number of spin-up states which can be used for further demagnetizing electron-phonon scatterings. Altogether, electron-magnon scatterings increase the phase space for demagnetizing electron-phonon scatterings, and therefore a combination of electron-phonon and electron-magnon scatterings may explain the experimentally observed demagnetization.

\section{General Issue 1: Transfer of Angular Momentum}

During ultrafast demagnetization at least part of the angular momentum of the electronic spin system is transferred to the lattice, i.e., to the angular momenta of lattice vibrations, by scatterings of electrons at these lattice vibrations. In all former papers on ultrafast demagnetization this transfer could not be calculated explicitly, because of the following reason. In these papers the authors described the lattice vibrations in terms of linearly polarized phonons (see, e.g., [9]) and calculated the change of the occupation numbers of them by scatterings at electrons. However, linearly polarized phonons do not have well-defined angular momenta, and therefore the change of the angular momentum of the lattice could not be determined from the calculated change of these occupation numbers. One will see in this section that the change of the angular momentum of the lattice can be calculated explicitly [22] by describing the lattice vibrations in terms of magnetoelastic spin-phonon modes [23].

Before introducing the magnetoelastic spin-phonon modes another point is discussed. The Hamiltonian

$$
\widehat{\mathrm{H}}=\widehat{\mathrm{H}}_{\text {electron }}+\widehat{\mathrm{H}}_{\text {phonon }}+\widehat{\mathrm{H}}_{\text {electron-phonon }} \text {, }
$$

which describes the scattering of electrons at lattice vibrations (either linearly polarized phonons or magnetoelastic spin-phonon modes) is not isotropic. The reason is that the

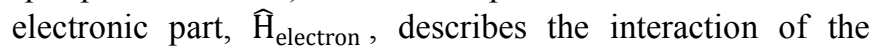
electrons with the lattice potential, which is not isotropic, and the phononic part, $\widehat{\mathrm{H}}_{\text {phonon, }}$ is described in terms of the force-constant matrix (see below) which is also not isotropic. The non-isotropy of the Hamiltonian has the result that the 
total angular momentum of the system is not conserved. Nevertheless, it is of course interesting to know how much of the angular momentum of the electronic spin system is transferred to the lattice during demagnetization when such a non-isotropic Hamiltonian is used. It may be argued that the universe is isotropic and thus that the total angular momentum is conserved. To treat this exactly, one had to take into account explicitly the coupling of the sample to the whole surroundings, but of course the Hamiltonian would be so complicated that the problem could not be attacked. However, a simplification arises because on the fs time scale a process in the magnetic system can lead to reactions only within a very limited spatial distance from the magnetic system, and this justifies the use of a non-isotropic Hamiltonian of type (11).

Now the magnetoelastic spin-phonon modes [23] are introduced. In an itinerant valence electron system like Ni and $\mathrm{Fe}$ the valence electrons build atomic magnetic moments, and these generate a magnetic field which may be described by a vector potential $\mathrm{A}$. The internal field exerts a Lorentz force on subunits of the atoms with charge $\mathrm{q}$, which is composed of the nuclear charges and the charges of the electrons in the closed electronic shells of the atoms [22]. When writing down an expression for the kinetic energy of the atom $n$ one has to take into account the vector potential A by replacing the normal momentum $\mathrm{p}_{\mathrm{n}}$ by its canonical conjugate momentum $\mathrm{p}_{\mathrm{n}}-\mathrm{q} / \mathrm{cA}$,

$$
\mathrm{E}_{\mathrm{kin}}=\sum_{\mathrm{n}}\left(\mathrm{M}_{\mathrm{n}}\right)^{-1}\left(\mathrm{p}_{\mathrm{n}}-\mathrm{q} / \mathrm{cA}\right)^{2}
$$

Here $M_{n}$ is the mass of the magnetic atom $n, c$ is the velocity of light. In harmonic approximation the potential energy is written in terms of the force-constant matrix $\underline{\underline{\phi}}$,

$$
\mathrm{E}_{\mathrm{pot}}=\mathrm{E}_{\mathrm{pot}}(\underline{\underline{\phi}})
$$

For such a system one can write down Newton's equations of motion for the displacements

$$
u_{n}\left(R_{n}, t\right)=\left(R_{n}(t)-R_{n}^{0}\right)
$$

of the positions $R_{n}(t)$ of the atoms from their equilibrium positions $\mathrm{R}_{\mathrm{n}}^{0}$. To solve these equations, the ansatz of a plane wave is made

$$
u_{n}\left(R_{n}(t)\right)=\frac{u_{0}}{\sqrt{M_{n}}} e_{q, \lambda} e^{i\left(q \cdot R_{n}-\omega_{q}, \lambda t\right)}
$$

Here $\mathrm{e}_{\mathrm{q}, \lambda}$ with $\lambda=1,2,3$ are the polarization vectors of the spin-phonon mode, $\mathrm{q}$ is its wavevector and $\omega_{\mathrm{q}, \lambda}$ is its frequency. Inserting this ansatz into the equations of motion yields a generalized eigenvalue problem, and the solution of this gives plane waves with complex polarization vectors $e_{q, \lambda}$.

Because of this complexity the magnetoelastic spin-phonon modes have well-defined angular momenta [23]. Therefore, Tsatsoulis et al. [22] were for the first time in the position to determine the change of the angular momentum of the lattice from the calculated change of the occupation numbers of the magnetoelastic spin-phonon modes due to their scatterings at electrons. For the scattering matrix elements the formalism was used which was described in section 3.2 for the scattering of electrons at linearly polarized phonons, just by replacing in the matrix elements the linearly polarized phonons by the spin-phonon modes.

By the formalism described above in [22] the transfer of the angular momentum from the electronic spin system to the lattice during ultrafast demagnetization for $\mathrm{Ni}$ and $\mathrm{Fe}$ was calculated. The result was that only few percent of the angular momentum of the electronic spin system is transferred to the lattice. The reason is that the nonisotropic Hamiltonian was used, so that the total angular momentum of the system is not conserved on the time scale of the ultrafast demagnetization.

The effect of the vector potential A on the vibrations of the neutral atoms is completely neglected in the normal theory of phonons. To repair this defect of the theory, one has to go to the magnetoelastic spin-phonon modes. As discussed above the advantage which one gets by this is that the spin-phonon modes have well-defined angular momenta (whereas the normal phonons do not have this feature). A weakness of the spin-phonon modes is that the Hamiltonian is not Hermitian and that therefore the set of spin-phonon modes is not complete. In principle one can consider a combination of momenta and coordinates to get a complete basis.

\section{General Issue 2: Effect of Electronic Correlations}

All former theories of ultrafast demagnetization used a conventional quantum theory for the spin-flip scatterings of electrons at quasiparticles, namely the time-dependent perturbation theory in the form of Fermi's golden rule for time-oscillating perturbations, in the case of ultrafast demagnetization given by the time oscillations of the quasiparticles. There are two preconditions for the use of Fermi's golden rule. First, it is a first-order theory, and by this it neglects the memory of the system to its former states, i.e., it makes a Markov approximation. Second, it is valid only for time scales which are considerably longer than the oscillation time of the periodic perturbation, because only then the Sinc-funtion of the perturbation theory can be approximated by Dirac's Delta functional which appears in Fermi's golden rule. However, the few hundred fs of ultrafast demagnetization are shorter than the oscillation times of typical quasiparticles. In all former theories Fermi's golden rule was used in combination with a band theory for the electronic states, at best in the sense of a Hubbard Hamiltonian. Thereby the electronic correlations in the sense of a density-matrix theory (which we will introduce below) are neglected. It has been shown that they have an essential effect on the ultrafast dynamics of nonmagnetic situations (see, e.g., [24]). Weng et al. [25] wanted to see whether their effect is also essential for the ultrafast dynamics of a magnetic situation, namely on the ultrafast de-and remagnetization after fs laser pulses. To investigate this, they used a quantum kinetic theory, the density-matrix theory [26], which takes into account these correlations. In [25] the ultrafast de-and remagnetization after laser pulses due to spin-flip scatterings of electrons at phonons were calculated, once by the combination of Fermi's golden 
rule with band theory and once with the density matrix theory. In their density-matrix theory they also made a Markov approximation and a long-time approximation by replacing the Sinc-function by Dirac's Delta functional. Therefore the comparison of the results of the two theories really yields the effect of electronic correlations. They used simple models for the single-electron energies $\varepsilon_{\mathrm{ik}}^{\mathrm{s}}$ where $\mathrm{k}$ is the electronic wave vector, $\mathrm{i}$ is the band index and $\mathrm{s}$ denotes the dominant spin character, simple models for the phonon energies and for the electron-phonon scattering matrix elements. The electronic correlations in the sense of a density-matrix theory are discussed in terms of the expectation values $\left\langle\widehat{C}_{k, i}^{\dagger} \widehat{C}_{k, j \neq i}\right\rangle$, where $\widehat{\mathrm{C}}_{\mathrm{k}, \mathrm{i}}^{\dagger}$ and $\widehat{\mathrm{C}}_{\mathrm{k}, \mathrm{i}}$ are operators which create and annihilate electrons in the state $|k, i\rangle$. These expectation values are nonzero only if the electrons show transitions between various bands $i$ and $j \neq i$, for instance by spin-flip scattering processes. In a Hubbard band theory for the electronic band structure there are also electronic correlations. They describe the effect of Coulomb interactions between the electrons, which has two contributions. First, the mean Coulomb interaction of an electron with all the other electrons, described by the Hartree potential which appears in a normal band theory, and second, the deviation of the real Coulomb interaction energy from this mean Coulomb interaction energy, which is discussed in terms of electronic correlations of type $\left\langle\widehat{\mathrm{C}}_{\mathrm{k}, \mathrm{i}}^{\dagger} \widehat{\mathrm{C}}_{\mathrm{k}, \mathrm{i}}\right\rangle$, and which appears in a Hubbard type band theory. These correlations are completely different from the above introduced electronic correlations in the sense of a density-matrix theory, which do not appear in a Hubbard-type band theory.

For the formalism of the density-matrix theory see [25]. Here just the very unexpected result is discussed. As discussed above, the electronic correlations in the sense of a density-matrix theory are essential for the ultrafast dynamics of a nonmagnetic situation. In contrast, it was found that their effect on the ultrafast dynamics of a magnetic situation, namely the ultrafast de-and remagnetization after fs laser pulses, is just marginal. This is a very important result from a fundamental point of view, interesting for all people working on ultrafast dynamics. It is also important from a practical point of view.

As discussed above, all former papers on ultrafast de-and remagnetization used a combination of Fermi's golden rule with a band theory, in which the electronic correlations in the sense of a density-matrix theory are neglected. The above discussed result justifies the use of this combined theory from the viewpoint of the neglect of electronic correlations.

\section{Conclusion}

In the present review we have shown that the ultrafast de-and remagnetization after fs laser pulses is caused by a complex interaction of light with quantum matter, and that its description requires very sophisticated and interesting theoretical methods. We hope that this review will initiate extensions of the theory in various ways. First, for the calculation of the transfer of angular momentum from the electronic spin system to the lattice on the time-scale of ultrafast dynamics, one could use a Hamiltonian which contains more degrees of freedom than just the magnetoelastic spin-phonon modes (as discussed in section 4, the set of spin-phonon modes is not complete). An example would be to include local elastic twists in the sample as reactions on local torques, twists that have a small amplitude but have angular momenta that add up in the whole sample. The related Hamiltonian would still be nonisotropic, so that the total angular momentum is still not conserved, but the inclusion of the local twists may change the results quantitatively. Second, it is desirable to perform a density-matrix theory without a Markov approximation and without using a long-time approximation. Furthermore, it would be interesting to study whether the effect of electronic correlations is marginal also for other contributions (not just for the contribution of electron-phonon scatterings) to ultrafast dynamics (e.g., contributions of electron-electron scatterings and electron-magnon scatterings and of a combination of electron-phonon and electron-magnon scatterings).

\section{Acknowledgements}

The authors are indebted to the department head, Gisela Schütz, who strongly supported their research on ultrafast de-and remagnetization after fs laser pulses.

\section{References}

[1] B. Koopmans, G. Malinowski, F. Dalla Longa, D. Steiauf, M. Fähnle, T. Roth, M. Cinchetti, and M. Aeschlimann, "Explaining the paradoxial diversity of ultrafast laser-induced demagnetization", Nat. Mater. 9, 259-265 (2010).

[2] A. Kirilyuk, A. V. Kimel, and T. Rasing, "Ultrafast optical manipulation of magnetic order", Rev. Mod. Phys. 82, 2731-2784 (2010).

[3] E. Beaurepaire, J.-C. Merle, A. Daunois, and J.-Y. Bigot, "Ultrafast Spin Dynamics in Ferromagnetic Nickel", Phys. Rev. Lett. 76, 4250-4253 (1996).

[4] M. Battiato, K. Carva, and P. Oppeneer, "Super-Diffusive Spin Transport as a Mechanism for Ultrafast Demagnetization", Phys. Rev. Lett. 105, 027203-027206 (2010).

[5] M. Shalaby, C. Vicario, and C. P. Hauri, "Low frequency terahertz-induced demagnetization in ferromagnetic Nickel", Appl. Phys. Lett. 108, 162903-162906 (2016).

[6] Y. Yafet, "g Factors and Spin-Lattice Relaxation of Conduction Electrons", in Solid State Physics, Vol. 14, edited by F. Seitz and D. Turnbull (Academic, New York), 1 ff (1963).

[7] B. Y. Mueller, A. Baral, S. Vollmar, M. Cinchetti, M. Aeschlimann, H. C. Schneider, and B. Rethfeld, "Feedback Effect during Ultrafast Demagnetization Dynamics in Ferromagnets", Phys. Rev. Lett. 111, 167204-167208 (2013).

[8] B. Y. Mueller, M. Haag, and M. Fähnle, "Ab-initio theory for ultrafast magnetization dynamics with a dynamic band structure", J. Magn. Magn. Mater. 414, 14-18 (2016). 
[9] C. Illg, M. Haag, and M. Fähnle, "Ultrafast demagnetization after laser irradiation in transition metals: Ab-initio calculations of the spin-flip electron-phonon scattering with reduced exchange splitting", Phys. Rev. B 88, 214404-1214404-10 (2013).

[10] L. Nordheim, "Zur Elektronentheorie der Metalle", Ann. Phys. 401, 607-640 (1931).

[11] C. Illg, B. Meyer, and M. Fähnle, "Frequencies and polarization vectors of phonons: Results from force constants which are fitted to experimental data or calculated ab initio", Phys. Rev. B 86, 174309-1-174314-6 (2012).

[12] C. Ederer, "Theorie magneto-optischer Effekte im Röntgenbereich und Anwendung auf niedrigdimensionale Systeme", PhD thesis, Max Planck Institute for Metals Research and University of Stuttgart (2003).

[13] O. K. Andersen and O. Jepsen, "Explicit First-Principles Tight-Binding Theory", Phys. Rev. Lett. 53, 2571-2574 (1984).

[14] Y. P. Perdew, and Y. Wang, "Accurate and simple density functional for the electronic exchange energy, Generalized Gradient Approximation", Phys. Rev. B 33, 8800-8802 (1986).

[15] Y. P. Perdew, and Y. Wang, "Accurate and simple analytic representation of the electron-gas correlation energy", Phys. Rev. B 45, 13244-13249 (1992).

[16] M. Haag, C. Illg, and M. Fähnle, "Theory of scatterings of crystal electrons at magnons", Phys. Rev. B 87, 214427- 1214434-8 (2013).

[17] O. Grotheer, 2002, "Ab-initio Berechnungen der Spinwellenspektren von Eisen, Kobalt und Nickel", PhD thesis, Max Planck Institute for Metals Research and University of Stuttgart (2002).

[18] E. Carpene, E. Mancini, C. Dallera, K. Brenna, E. Puppin, and
S. De Silvestri, "Dynamics of electron-magnon interaction and ultrafast demagnetization in thin iron films", Phys. Rev. B 78, 174422-1-174422-6 (2008).

[19] M. Haag, C. Illg, and M. Fähnle, "Role of electron-magnon scatterings in ultrafast demagnetization", Phys. Rev. B 90, 014417-1-014417-6 (2014).

[20] A. Manchon, Q. Li, L. Xu, and S. Zhang, "Theory of laser-induced demagnetization at high temperatures", Phys. Rev. B 85, 064408-1-064416-9 (2012).

[21] A. J. Schellekens and B. Koopmans, "Comparing Ultrafast Demagnetization Rates Between Competing Models for Finite Temperature Magnetism", Phys. Rev. Lett. 110, 214204-214208 (2013).

[22] T. Tsatsoulis, C. Illg, M. Haag, B. Y. Mueller, L. Zhang, and M. Fähnle, "Ultrafast demagnetization after femtosecond laser pulses: Transfer of angular momentum from the electron system to magnetoelastic spin-phonon modes", Phys. Rev. B 93, 134411-1-134417-7 (2016).

[23] L. Zhang and Q. Niu, "Angular momentum of phonons and the Einstein-de Haas effect", Phys. Rev. Letters 112, 085503-085507 (2014).

[24] M. Joschko, M. Woerner, T. Elsaesser, E. Binder, T. Kuhn, R. Hey, H. Kostial, and K. Ploog, "Heavy-Light Hole Quantum Beats in the Band-to-Band Continuum of GaAs observed in 20 Femtosecond Pump-Probe Experiments", Phys. Rev. Lett. 78, 737-740 (1997).

[25] W. Weng, J. Briones, N. Teeny, B. Y. Mueller, M. Haag, T. Kuhn, and M. Fähnle, "Unexpectedly marginal effect of electronic correlations on ultrafast demagnetization after femtosecond laser pulses", Phys. Rev. B 95, 224439-1224443-4 (2017).

[26] D. Ter Haar, "Theory and applications of the density matrix", Reports on Progress in Physics 24, 304-362 (1961). 